\title{
What can repetition, reading and naming tell us about Jargon Aphasia?
}

Article

Accepted Version

Pilkington, E., Sage, K., Saddy, D. and Robson, H. (2019)

What can repetition, reading and naming tell us about Jargon Aphasia? Journal of Neurolinguistics, 49. pp. 45-56. ISSN 0911-6044 doi:

https://doi.org/10.1016/j.jneuroling.2018.08.003 Available at https://centaur.reading.ac.uk/78781/

It is advisable to refer to the publisher's version if you intend to cite from the work. See Guidance on citing.

To link to this article DOI: http://dx.doi.org/10.1016/j.jneuroling.2018.08.003

Publisher: Elsevier

All outputs in CentAUR are protected by Intellectual Property Rights law, including copyright law. Copyright and IPR is retained by the creators or other copyright holders. Terms and conditions for use of this material are defined in the End User Agreement.

\section{www.reading.ac.uk/centaur}

\section{CentAUR}

Central Archive at the University of Reading 
Reading's research outputs online 
What can repetition, reading and naming tell us about Jargon Aphasia?

Emma Pilkington $^{\mathrm{a}^{*}}$, Karen Sage ${ }^{\mathrm{b}}$, James Douglas Saddy ${ }^{\mathrm{a}}$, and Holly Robson ${ }^{\mathrm{a}}$

${ }^{a}$ School of Psychology and Clinical Language Sciences, University of Reading, Reading, UK.

${ }^{b}$ Department of Allied Health Professions, Sheffield Hallam University, Sheffield, UK.

*School of Psychology and Clinical Language Sciences, University of Reading, Reading, UK, RG67BE. E.c.pilkington@pgr.reading.ac.uk

Word count: $\mathbf{5 7 1 3}$

For Submission to: Journal of Neurolinguistics

Date Submitted: 20/03/2018

Date of revised submission 1: 13/07/2018

Date of revised submission 2: 14/08/2018 


\section{What can repetition, reading and naming tell us about Jargon Aphasia?}

\section{Abstract}

3 Jargon Aphasia is an acquired language disorder characterised by high proportions of

4 nonword error production, rendering spoken language incomprehensible. There exist two

5 major hypotheses relating to the source of nonword error; one implicates disruption to

6 phonological processing and the other suggests both phonological and lexical contributions.

7 The lexical sources are described as failure in lexical retrieval followed by surrogate

8 phonological construction, or a lexical selection error further compounded by phonological

9 breakdown. The current study analysed nonword error patterns of ten individuals with fluent

10 Neologistic Jargon aphasia in word repetition, reading and picture naming to gain insights into the contributions of these different sources. It was predicted that, if lexical retrieval deficits contribute to nonword production, naming would produce a greater proportion and severity of nonword errors in comparison to repetition and reading, where phonology is present and additional sub-lexical processing can support production. Both group and case series analyses were implemented to determine whether quantity and quality of nonwords differed across the three production tasks. Nonword phoneme inventories were compared against the normative phoneme distribution to explore whether phonological production takes place within a typically organised, lexically constrained system. Results demonstrated fewer nonword errors in naming and a tendency for nonwords in naming to be characterised by lower phonological accuracy. However, nonwords were, for the most part, constructed with reference to target phonological information and, generally, nonword phonological production patterns adhered to the statistical properties of the learned phonological system. While a subset of the current group demonstrated very limited lexical processing capacity which manifested as nonword errors in naming being most disrupted, overall the results suggest that nonwords are largely underpinned by some degree of successful lexical retrieval 
26 and implicate phonological sources, which manifest more severely when production is

27 accomplished via nonlexical processing routes.

28 Keywords: Jargon aphasia; nonword; neologism; Phonological Overlap Index (POI); word 29 production 


\section{Introduction}

\subsection{Nonword production}

32

Jargon aphasia is a form of acquired language impairment characterised by nonword errors in spoken production. Nonwords occur across all output tasks, and the presence of nonwords within connected speech renders spoken production incomprehensible (Marshall, 2006). Efforts to elicit nonword errors in neurologically healthy speakers have applied external manipulations such as phonological priming and response pressure to word production tasks. However, real words, i.e. words with existing conceptual and lexical representations, continue to dominate output, whilst nonword errors are rarely realised (Baars, Motley, \& MacKay, 1975; Goldrick \& Blumstein, 2006; Vitevitch, 2002). This failure to prime nonword errors to the same extent at which they are observed within the Jargon aphasia population limits understanding of the mechanism(s) underlying nonword production and hinders the development of hypotheses attempting to explain how such production comes to dominate in a form of acquired language impairment.

Despite this, there exist a number of theoretical accounts pertaining to nonword error generation, mostly derived from studies of picture naming in clinical populations. The most widely accepted hypothesis postulates that nonwords stem from a single impairment source a deficit in phonological encoding. The phonological encoding account states that deficient activation of target phonological segments for output allows alternative phonemes to compete and intrude, giving rise to non-target phonology in production (Kertesz \& Benson, 1970). Nonwords with high proportions of target phonology (paraphasia, e.g. village, /lividz/) are hypothesised to arise through mild disruption to this stage of phonological processing, whereas errors with little or no target phonology (neologism, e.g. tribute, /krarbri:/) are thought to follow more significant disruption during segment selection and organisation. By 
this hypothesis paraphasias and neologisms occupy opposite ends of a single continuum of nonword severity and the majority of nonwords fall somewhere in between and contain moderate degrees of target phonology (Dell, Schwartz, Martin, Saffran, \& Gagnon, 1997; Olson, Halloran, \& Romani, 2015; Olson, Romani, \& Halloran, 2007; Schwartz, Wilshire, Gagnon, \& Polansky, 2004). However, some case studies document evidence that challenge this hypothesis, reporting individuals who produce significant proportions of nonwords that share very little or no target phonology and high proportions of non-target phonological segments. Such observations have given rise to alternative hypotheses which propose that nonwords stem from a dual impairment in lexical and phonological processing. Under such hypotheses, severe neologisms are underpinned by a separate or additional lexical deficit. One such hypothesis suggests that severe distortions occur when the lexical representation belonging to the target word is unable to be retrieved and subsequently a surrogate phonological string is assembled for output, without reference to the target lexical representation (Buckingham, 1977; 1990; Butterworth, 1979, 1992; Butterworth, Swallow, \& Grimston, 1981; Buckingham, 1977). A complementary hypothesis suggests that severe neologisms are formed by compound errors, in which erroneous lexical selection is followed by faulty phonological encoding (Schwartz, Wilshire, Gagnon, \& Polansky, 2004). Evidence for the single and dual source hypotheses can be examined by exploring the phonological accuracy of nonwords and the distribution of this accuracy. A single phonological locus (one source) would generate a majority of errors containing moderate levels of target phonology, since nonword construction follows appropriate lexical retrieval. Additionally, there would be a comparative scarcity of errors with few/significant portions of target phonology, thus eliciting a normal distribution of accuracy (Olson et al., 2007; 2015; Pilkington et al., 2017; Schwartz et al., 2004). A separate lexical deficit would generate an independent error population, characterised by a significant proportion of responses containing chance levels of 
target phonology, secondary to surrogate phonological usage in the absence of a specified lexical target or phonologically distorted lexical errors. The coexistence of lexical and phonological error sources would be reflected in a bimodal distribution of accuracy and has been illustrated in some case studies of Jargon individuals (Buckingham \& Kertesz, 1976; Kohn et al., 1996).

\subsection{Production task differences}

An alternative approach to differentiating between the single and dual source hypotheses is to analyse production patterns across separate output tasks which are characterised by different lexical and phonological processing demands. Specifically, picture naming requires independent semantic and lexical retrieval prior to phonological encoding, such that errors arising through lexical processes, either default phonological selection secondary to lexical failure, or compound lexical and phonological errors should be more likely in this task, and so a greater number of nonword errors should occur, if a lexical source exists. Furthermore, given that some of these errors are characterised by lexical selection errors/failures, the quality of nonword errors in naming should be affected, with lower accuracy in phonological production expected (Olson et al., 2007). Reading and repetition can be supported by both lexical and nonlexical processes concurrently and so fewer nonwords should be observed in these tasks, since nonlexical processing can support and facilitate production, thereby allowing production to be accomplished with less weight on lexical retrieval (Coltheart, Curtis, Atkins \& Haller, 1993; Roelofs, 2004). Since phonological encoding is common in all three production tasks, a single phonological locus would elicit similar numbers of nonword errors across tasks. However, previous production task comparisons in Jargon aphasia have produced inconsistent results. The nature and number of nonword errors produced in repetition, reading and naming has been observed to be relatively consistent in some individuals with Jargon aphasia (Moses, Nickels, \& Sheard, 2007; Olson et al., 2007; 2015) 
whereas other cases have presented with greater nonword errors in naming than in other production tasks including reading and repetition (Ackerman and Ellis, 2007; Corbett, Jeffries, \& Lambon-Ralph, 2008; Moses, Nickels, \& Sheard, 2004). Importantly, much of this previous evidence is derived from single case studies or includes individuals with mixed behavioural profiles and relatively mild Jargon deficits, limiting the applicability and relevance of these conclusions to individuals with more severe production deficits.

\subsection{Jargon phonological inventories}

Further evidence into the source of nonword errors can be gained by exploring the phonological inventories of individuals with Jargon aphasia. Phonological inventories, the frequency of occurrence of each phonological segment within an individual's nonword inventory, reflects the statistical properties of the phonological system and suggests whether a lexical influence remains over production, as the phonological segment selection is inherently linked and influenced by a word's lexical representation. A number of Jargon aphasia cases have been identified in which individuals present with idiosyncratic phonological usage. This indicates that the phonological system does not retain its statistical structure and that nonwords may not be constrained by lexical processing and supporting the total lexical retrieval failure hypothesis (Butterworth, 1979; Eaton, Marshall, \& Pring, 2010; Moses et al., 2004). Originally, such patterns were proposed to arise from a neologism generating device or mechanism (Buckingham, 1990; Butterworth, 1979). However, an alternative interpretation is that idiosyncratic phonological useage arises through long term disruption to phonological encoding, which distorts the phonological system and the frequency at which each individual segment resides (Eaton, Marshall, \& Pring, 2010; Moses et al., 2004; Robson, Pring, Marshall, \& Chiat, 2003).

\subsection{The current study}


In the current study, we apply these methodological approaches to a case series of individuals with Neologistic Jargon aphasia to draw inferences regarding the source(s) of impairment and functioning of the phonological system. Single word naming, reading and repetition data were collected from ten participants with Jargon aphasia. We analyse the prevalence of nonword errors across the three separate production tasks and examine the phonological accuracy of nonword responses to understand whether nonword errors manifest differently in the separate tasks. We also explore whether phonological segment frequency within nonwords conforms to typical English frequencies to determine whether production is constrained by a typically organised lexico-phonological processing system.

\section{Methods}

\subsection{Participants}

Ethical approval for this project was gained from the North West NHS Research Ethics Committee. Ten individuals (one female; age $\overline{\mathrm{x}}=69$ years, $\sigma=10.2$ years; time post onset $\overline{\mathrm{x}}$ $=19$ months, $\sigma=22.15$ months) with Jargon aphasia are reported. Data were collected by the last author between 2009 - 2011 and all participants gave informed consent. All ten individuals produced high proportions of neologistic and/or paraphasic errors, with fluent speech and impaired single word comprehension (see Table 1). All ten individuals were classified as having Wernicke's Aphasia at the time of data collection, according to the Boston Diagnostic Aphasia Examination (Goodglass, Kaplan, \& Barresi, 2001). 

percentile results.

\section{BDAE percentile scores}

\begin{tabular}{|c|c|c|c|c|c|c|c|}
\hline $\begin{array}{c}\mathrm{Pt} \\
\text { code }\end{array}$ & $\begin{array}{c}\text { Age } \\
\text { (years) }\end{array}$ & Sex & $\begin{array}{c}\text { Time post } \\
\text { onset } \\
\text { (months) }\end{array}$ & Comprehension & Fluency & $\begin{array}{l}\text { Word } \\
\text { repetition }\end{array}$ & $\begin{array}{l}\text { Sentence } \\
\text { repetition }\end{array}$ \\
\hline p1 & 70 & $\mathrm{M}$ & 42 & 45 & 100 & 15 & 40 \\
\hline $\mathrm{p} 2$ & 60 & M & 5 & 6.5 & 84 & 5 & 10 \\
\hline p3 & 59 & M & 6 & 17 & 100 & 10 & 30 \\
\hline $\mathrm{p} 4$ & 74 & M & 6 & 12 & 51 & 10 & 15 \\
\hline p5 & 64 & M & 6 & 10 & 68 & 15 & 15 \\
\hline p6 & 77 & M & 24 & 40 & 90 & 5 & 45 \\
\hline $\mathrm{p} 7$ & 78 & $\mathrm{~F}$ & 72 & 5 & 68 & 5 & 15 \\
\hline p8 & 86 & $\mathrm{M}$ & 13 & 10 & 80 & 5 & 10 \\
\hline p9 & 53 & $\mathrm{M}$ & 7 & 15 & 68 & $<1$ & $<1$ \\
\hline p10 & 73 & M & 6 & 3 & 63 & $<1$ & $<1$ \\
\hline
\end{tabular}

Note. Participants ordered by the total number of nonwords produced across the three production tasks from fewest (p1) to highest (p10).

\subsection{Tasks}

155 Participants undertook three single word production tasks - picture naming, reading and repetition. The picture naming test from the Cambridge Semantic Battery (Adlam, Patterson, Bozeat, \& Hodges, 2010) consisted of 64 black and white line drawings from the Snodgrass and Vanderwart set. Reading and repetition tests were 80-item subtests from the PALPA (Psycholinguistic Assessment of Language Processing in Aphasia, subtests 9 and 31: Kay, Lesser, \& Coltheart, 1996). To make the naming, reading and repetition tests numerically equivalent, a subset of 64 PALPA items were selected based on frequency ratings from NWatch (Davis, 2005) and the MRC psycholinguistic database (Coltheart, 1981). The repetition and reading sets included the same 64 target items (see Appendix 1) which had a 
mean frequency of $47.98(\sigma=1.40)$, mean familiarity $512.245(\sigma=69.96)$, mean imageability $431(\sigma=175.99)$, average number of letters $5.89(\sigma=1.40)$, mean number of phonemes $5,(\sigma$ $=1.49)$ and average syllable number $2.03(\sigma=0.76)$. The picture naming items had a similar mean frequency $(\overline{\mathrm{x}}=28.37, \sigma=56.60, \mathrm{t}(109)=1.945, p=.0543)$, familiarity $(\overline{\mathrm{x}}=514.02, \sigma=$ 73.66, $\mathrm{t}(107)=0.128, p=.898)$, imageability $(\overline{\mathrm{x}}=396, \sigma=291.10, \mathrm{t}(126)=0.807, p=$ $0.421)$, letter number $(\overline{\mathrm{x}}=6.17, \sigma=2.16, \mathrm{t}(126)=0.874, p=.384)$, phoneme number $(\overline{\mathrm{x}}=$ $4.918, \sigma=1.85, \mathrm{t}(126)=0.103, p=.785)$ and syllable number $(\overline{\mathrm{x}}=1.90, \sigma=0.80, \mathrm{t}(126)=$ $0.914, p=.359)$ to the repetition/reading tasks.

\subsection{Recording and error coding}

Responses were transcribed into DISC symbols (1:1 phoneme: symbol correspondence, i.e. $\mathrm{IPA}=[\mathrm{i}:]$, DISC $=[\mathrm{i}]) ;$ to enable automated data extraction via Microsoft excel. When multiple responses were given, the final complete utterance was accepted. Correct responses were identified, all non-lexical responses were labelled as nonwords, and remaining errors were grouped together.

\subsection{Analyses}

\subsubsection{Group error prevalence}

For each participant, the number of correct responses, nonword errors and other error types were counted. The number of nonwords observed from each participant on each production task (repetition, reading, naming) was entered into a one way repeated measures ANOVA to examine whether the number of nonword errors differed across repetition, reading and naming at the group level.

\subsubsection{Phonological accuracy of nonwords}

\subsubsection{Observed accuracy}


The Phonological Overlap Index (POI) (number of phonemes shared between response and target $\mathrm{x} 2) /($ total phonemes in target + total phonemes in response) (Bose, 2013; Schwartz et al., 2004) was calculated for each nonword. This calculation assigns responses which contain all appropriate target phonemes a value of one, and responses which contain no target segments a value of zero. When all appropriate phonemes are selected, irrespective of their order a nonword would attain a value of one (e.g. village, /lividz/). A one way repeated measures ANOVA was used to determine whether phonological accuracy (POI) differed across repetition, reading and naming. To determine whether phonemes were accurately encoded at the individual level, average POI values for each participant on each production task were compared against a chance level of accuracy via a bootstrapping procedure.

\subsubsection{Chance phonological accuracy}

A chance phonological overlap (POI) statistic represents the degree to which any target response pairing is likely to share phonology. This statistic quantifies the extent to which a nonword will overlap with a target if it were constructed without reference to target phonology and reflects the degree of accuracy expected from random phonological assembly. To calculate chance, all nonword responses produced by the ten individuals within a specific task were collated, along with their corresponding target words. The response and target sets were randomly shuffled, thereby reassigning each nonword error to a new target word. The number of nonwords produced by each individual in each modality was used to determine how many randomly paired responses to sample from the chance sample; for example where p10 produced 63 nonwords in repetition, 63 random pairings were sampled to derive an individual null distribution. The POI for each new target-nonword pair was calculated and the average across these pairings was derived. This process was repeated 1000 times to yield 
211 statistic for each individual per production task. Confidence intervals for the null distribution

212 were obtained by identifying the chance values observed at the top and bottom $2.5 \%$.

\section{2.4.2.3 Phonological accuracy distributions}

214 Individual POI distributions were analysed using the Shapiro Wilk test of normality.

215 Normally distributed POI data are proposed to reflect a single phonological nonword error

216 source. A dual error source is proposed to produce a bimodal distribution. Histograms were

217 visually inspected to assess whether bimodal distributions occurred if testing indicated

218 violation of normality. Where normality was violated, histograms were interpreted to

219 determine whether a bimodal distribution was observed, indicating separate nonword error

220 sources underpinned by failed lexical retrieval and phonological error, or erroneous lexical

221 selection followed by phonological distortion.

\section{$222 \quad 2.4 .3$ Phoneme frequency distributions}

223 The frequency of each phoneme in each participant's nonword error set was calculated and compared against the expected phoneme frequency in English, as reported in Denes (1963). Nonword errors were collated across production task to provide sufficient data to run this analysis; focusing on phonemic diversity on a single data point/collection time would make this analysis vulnerable to perseveration and may falsely indicate a distorted phonological inventory. Each individual's phoneme frequency distribution was compared against the normative distribution, using a type two Kolmogorov Smirnov test. 


\subsection{Group error prevalence}

232 Table 2 reports the number of nonword errors produced by each of the ten participants across

233 repetition, reading and naming. A one way repeated measures ANOVA was used to

234 determine whether numbers of nonword error differed across task (repetition, reading,

235 naming). There was a significant effect of production task on the numbers of nonword

236 production $\left(\mathrm{F}(2,18)=4.840, p=.021, \eta p^{2}=.350\right.$, see Figure 1$)$, and post hoc - pairwise

237 comparisons tests applying Bonferroni correction identified that picture naming elicited

238 significantly fewer nonwords than reading $(p=.008)$. Additional pairwise comparisons did

239 not identify any further differences $(p \geq .227)$.

241 Table 2: The number of correct responses, nonwords and other errors produced by each

242 participant across repetition, reading and naming.

\begin{tabular}{cccccccccc}
\hline & \multicolumn{3}{c}{ Repetition } & \multicolumn{3}{c}{ Reading } & \multicolumn{3}{c}{ Naming } \\
\hline & Correct & Nonwords & Other & Correct & Nonwords & Other & Correct & Nonwords & Other \\
\hline p1 & 30 & 25 & 9 & 38 & 21 & 5 & 46 & 9 & 9 \\
p2 & 18 & 18 & 28 & 22 & 26 & 16 & 28 & 15 & 21 \\
p3 & 32 & 16 & 16 & 20 & 39 & 5 & 31 & 22 & 11 \\
p4 & 32 & 22 & 10 & 6 & 45 & 13 & 16 & 29 & 19 \\
p5 & 5 & 57 & 2 & 20 & 32 & 12 & 12 & 15 & 37 \\
p6 & 17 & 36 & 11 & 11 & 44 & 9 & 21 & 33 & 10 \\
p7 & 4 & 50 & 10 & 9 & 49 & 6 & 11 & 20 & 33 \\
p8 & 4 & 44 & 16 & 7 & 51 & 6 & 9 & 41 & 14 \\
p9 & 4 & 51 & 9 & 2 & 54 & 8 & 7 & 37 & 20 \\
p10 & 1 & 63 & 0 & 11 & 50 & 3 & 2 & 61 & 1 \\
\hline
\end{tabular}

$243 *$ Other $=$ semantic, , ormal, mixed, circumlocution, unrelated and non-response collated. 


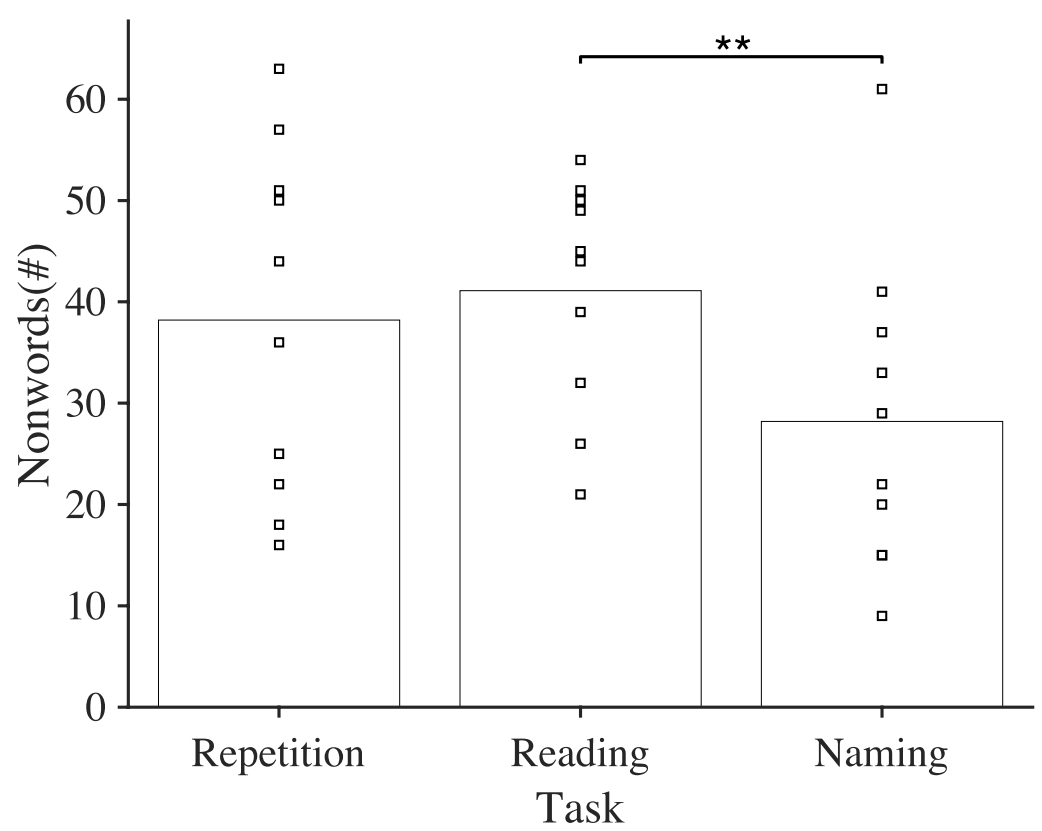

253 Figure 1 Title: Nonword Production in Repetition, Reading and Naming.

254 Figure 1 Legend: Bar chart displays the mean number of nonword responses in each task.

255 Individual markers indicate participant nonword numbers.

\subsection{Phonological accuracy of nonwords}

\subsubsection{Observed phonological accuracy}

259 The accuracy of all nonword errors was measured using the Phonological Overlap Index

260 (POI) calculation, thereby assigning values between 0 and 1 to all nonwords, with a value of

261 one reflecting complete phonological overlap between a nonword and target word pair. A

262 repeated measures ANOVA was used to compare average POIs across the three output tasks.

263 The ANOVA identified a significant effect of task on phonological accuracy $(\mathrm{F}(2,18)=$ 5.665, $p=.012, \eta p^{2}=.386$, see Figure 2); with post-hoc, Bonferonni corrected, pairwise 


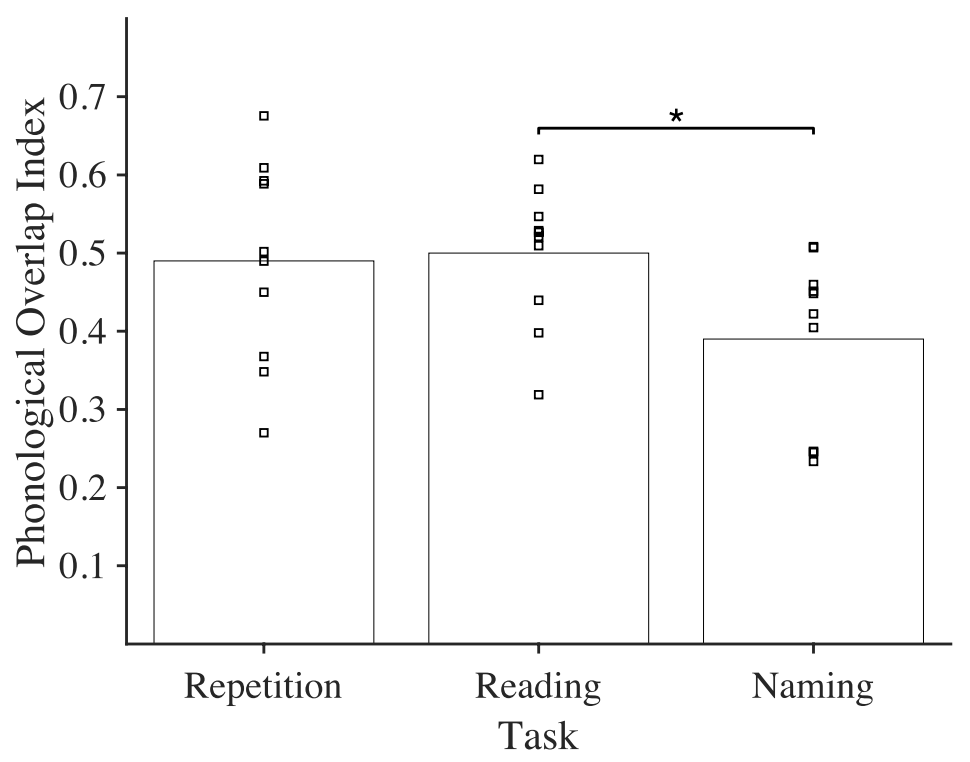

275

276 Figure 2 Title: Phonological Overlap Index in Repetition, Reading and Naming.

277 Figure 2 Legend: Bar chart displays mean Phonological Overlap Index (POI) of nonword errors in each production task. Individual markers represent participant POI means.

280 For each participant the average POI was calculated for all nonwords in each separate

281 production task and compared against a chance value of phonological accuracy using a

282 bootstrapping procedure. In repetition all ten individuals produced nonwords that contained greater degrees of target phonology than predicted by chance (POI $\overline{\mathrm{x}} \geq 0.270, p \leq .002$; see Figure 3a). The same pattern was observed in reading (POI $\overline{\mathrm{x}} \geq 0.318, p \leq .001$; see Figure 3b). In picture naming, $\mathrm{p} 4$ produced target phonology at chance levels (POI $\overline{\mathrm{x}}=0.245, p=$ 0.54; see Figure 3c). The remaining nine individuals produced target phonology at greater 287 than the chance prediction (POI $\overline{\mathrm{x}} \geq 0.247, p \leq .035$; see Figure $3 \mathrm{c}$ ). 
A

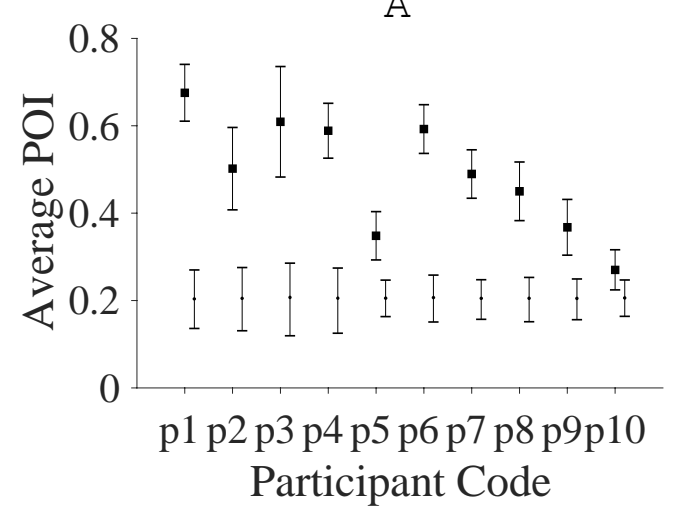

Participant Code

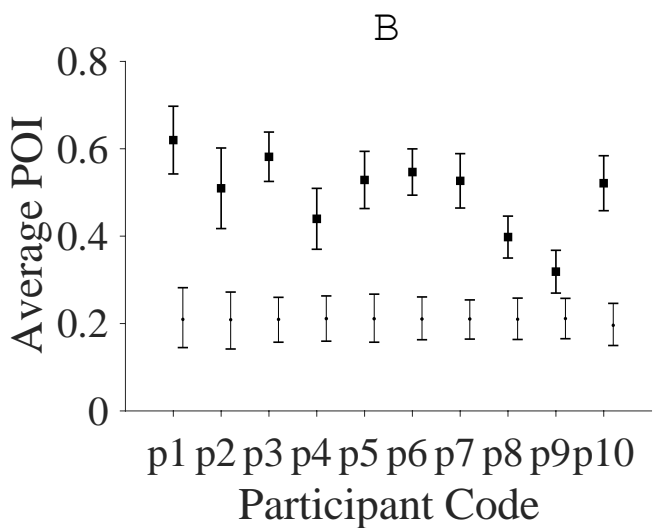

C

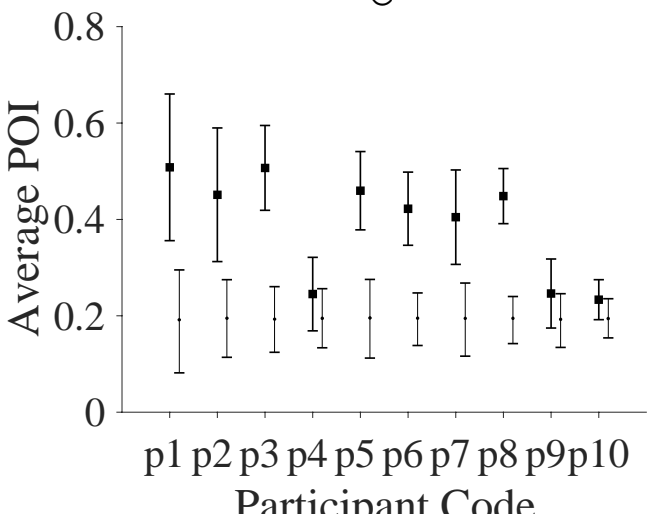

I Observed

Chance

Figure 3: Participant Phonological Overlap Index vs. Chance Phonological Overlap Index nonwords produced in Repetition (A), Reading (B) and Picture Naming (C). Error bars indicate $95 \%$ confidence intervals.

\subsubsection{Accuracy distributions}

The Shapiro Wilk test was used to examine whether nonword accuracy (POI) spread conformed to a normal distribution, thereby suggesting a single phonological locus of nonword error. The POI distributions exhibited by seven individuals $(\mathrm{p} 1,2,3,5,6,7,8)$ either conformed to a normal distribution $(p \leq 0.077)$ or followed a negative skew, indicating a tendency towards higher target overlap (a greater proportion of nonwords observed above the mean, see Table 3 marked $^{\mathbf{\Delta}}$ ). The POI accuracy distribution for $\mathrm{p} 4$ did not follow a normal distribution in naming $(p=0.013$, skewness $=0.529$, Figure 4D); $\mathrm{p} 9$ also exhibited a normality violation in naming $(p=0.003$, skewness $=0.721$, Figure $4 \mathrm{C}) ; \mathrm{p} 10$ violated the 
normal distribution in repetition $(p=0.005$, skewness $=0.620$, Figure $4 \mathrm{~B})$ and in naming $(p=$ 3030.004 , skewness $=0.258$, Figure 4A). Visual inspection of these histograms indicate a heavy 304 skew towards lower phonological accuracy with a graded increase in accuracy from zero, 305 rather than a bimodal distribution (see Figure 4).

306 Table 3: $p$ statistic from Shapiro Wilk normality test of POI distribution.

\begin{tabular}{cccc}
\hline & Repetition & Reading & Naming \\
\hline p1 & 0.092 & 0.204 & 0.294 \\
p2 & 0.757 & 0.090 & 0.190 \\
p3 & 0.244 & 0.263 & 0.608 \\
p4 & 0.155 & 0.187 & $0.013 \bullet$ \\
p5 & 0.115 & 0.136 & 0.452 \\
p6 & $0.020 \boldsymbol{\Delta}$ & 0.153 & 0.625 \\
p7 & 0.067 & $0.039 \boldsymbol{\triangle}$ & 0.077 \\
p8 & 0.217 & 0.761 & 0.663 \\
p9 & 0.109 & 0.082 & $0.003 \bullet$ \\
p10 & $0.005 \bullet$ & 0.267 & $0.004 \bullet$
\end{tabular}

307

Symbol Key: ${ }^{\boldsymbol{\Delta}}$ negative skew (majority of POI fell above the mean); ${ }^{\bullet}$ positive skew. 

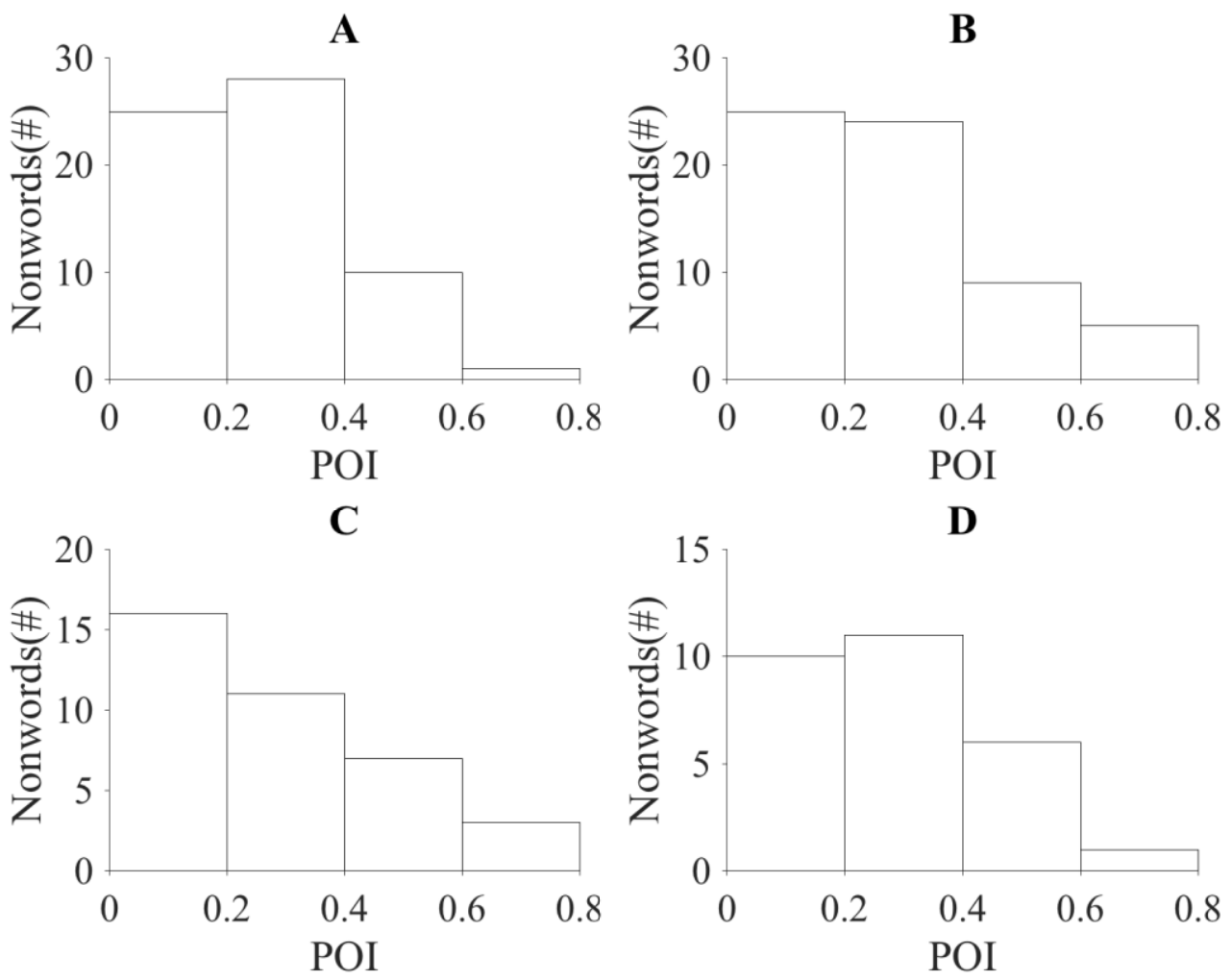

311 Figure 4: Phonological Overlap Index distributions when normality violated. (A) p10

312 Naming, (B) p10 Repetition, (C) p9 Naming, (D) p4 Naming.

\subsection{Phoneme frequency distributions}

315 The Kolmogorov Smirnov Two-sample test (KS2) was used to identify whether the nonword 316 phoneme inventory of each individual participant conformed to English norms (Dene, 1963).

317 To ensure sufficient data for this analysis, nonword phonemes were collapsed across

318 production task and overall prevalence of each phoneme was calculated as a percentage. The 319 KS2 test demonstrated that all ten individuals distributed phonemes in line with the expected normative pattern ( $p \geq 0.076$; see Table 4 for full results). Figure 5 depicts the phoneme frequency distributions for each Jargon participant, with box plots reflecting negatively skewed distributions similar to that of English norms. 
324 Table 4: $Z$ statistic and $p$ value from Kolmogorov Smirnov two (KS2) test comparing 325 normative and individual nonword phoneme frequency distributions.

\begin{tabular}{ccc}
\hline & KS Z $^{\mathrm{a}}$ & $P$ \\
\hline $\mathrm{p} 1$ & 1.173 & 0.128 \\
$\mathrm{p} 2$ & 1.386 & 0.043 \\
$\mathrm{p} 3$ & 0.853 & 0.461 \\
p4 & 0.959 & 0.316 \\
p5 & 1.279 & 0.076 \\
p6 & 0.853 & 0.461 \\
p7 & 1.173 & 0.128 \\
p8 & 1.279 & 0.076 \\
p9 & 1.173 & 0.128 \\
p10 & 0.853 & 0.461
\end{tabular}



330 Figure 5: Phoneme frequency distributions for English norms and participants. 


\subsection{Group error prevalence}

333 This study examined the nonword error patterns produced on single word repetition, reading and picture naming tasks in a group of ten people with Jargon aphasia. Current hypotheses propose that nonwords arise through either a single, phonological source or a dual impairment in lexical and phonological processing. A single phonological source predicts that a similar proportion of nonword errors will be produced across the different production tasks, since the phonological encoding requirements are similar (Olson et al., 2007; 2015). A dual source predicts that a greater proportion of nonword errors will be observed in naming than in reading and repetition, as naming weighs more heavily on lexical processing and cannot utilise sub-lexical processing to support production in the event of deficient lexical information (Coltheart et al., 1993; Moses et al., 2004; Nozari, Kitteridge, Dell, \& Schwartz, 2010; Olson et al., 2015). Results from the current study did not clearly conform to either of these patterns. Instead there were higher numbers of nonword errors in reading (statistically) and repetition (numerically) than in naming. Nevertheless, this result aligns best with the single phonological source hypothesis, in that more nonwords were produced in tasks with greater focus on phonological processing. Tasks which increased focus on lexico-semantic processing reduced the likelihood of nonword production. These results conflict with previous single case studies which have identified greater neologistic or error production impairments in Jargon naming (Ackerman \& Ellis, 2007; Moses et al., 2004; Corbett et al., 2008) and are inconsistent with patterns observed in the aphasia population generally where repetition tends to be more accurate than naming (Nozari et al., 2010). A significant proportion of this evidence comes from computational modelling studies which have described nonword production patterns primarily in naming and attempted to explain error patterns in other production tasks based on the naming models. The fewer numbers of nonword errors produced to tasks involving non-lexical processing components (e.g. 
repetition) are accounted for by recruitment of nonlexical processing routes which make use of surface word graphemes/phonemes and which can compensate for weak lexical route processing and bolster production accuracy (Dell et al., 1997; Hanley, Dell, Kay, \& Baron, 2004; Nozari et al., 2010). Picture naming, where nonlexical information is not available, lacks this additional boost and so is more likely to elicit errors. Closer examination of the cases within computational modelling studies (e.g. Nozari et al., 2010) demonstrate that individuals with poor language comprehension abilities such as that observed in Jargon aphasia, for example, those with Wernicke's aphasia, do not clearly conform to this dual route prediction and that these individuals produce error rates that are more equally balanced across the different production tasks; a pattern that is consistent with a subset of participants in the current group.

However, 4 participants (p1, p5, p7 and p9) produced more nonwords on both repetition and reading than in naming (similar trends were also observed in 3 other individuals, see Table 2), suggesting that dual route processing is not consistently operational in this sub set of individuals. The pattern exhibited by these 4 participants can, however, still be explained within existing frameworks of naming and repetition. Studies examining the balance between lexical and nonlexical processing in tasks such as reading and repetition have indicated differential routing patterns dependent on the person's ability to comprehend and recognise words (Nozari \& Dell, 2013). Individuals with greater lexical-semantic comprehension abilities favour the lexical processing route and make use of this for accomplishing tasks such as auditory repetition. People whose lexical comprehension and recognition are more severely impaired are pushed towards nonlexical processing as an alternative, since subsequent lexically motivated processing cannot proceed without sufficient lexical-word activation. All individuals in the current study had a diagnosis of Wernicke's aphasia and, consequently, severe impairments in analysing and processing input phonology, 
and comorbid impairments in lexico-semantic processing and comprehension (Robson, Sage, \& Lambon Ralph, 2012). In the current group, it is likely that impairments in language comprehension limit participant ability to access and use the lexical-semantic pathway to support production, thereby increasing reliance on surface level (nonlexical) information in tasks where this is possible (Nozari \& Dell, 2013). Additionally, the ability to decipher input phonology is significantly impaired in Wernicke's aphasia. Therefore, activation of target phonology from the nonlexical route will be severely disrupted, which will increase the likelihood of observing a nonword. This pattern of processing can explain the greater number of nonword errors observed in repetition/reading in comparison to picture naming.

\subsection{Case series analyses}

The single source interpretation is challenged by the finding that the phonological accuracy of nonword errors (target-error overlap, measured by the POI) was lower in naming than in reading and repetition. This could be taken as evidence for an additional lexical impairment contributing to nonwords either through complete lexical retrieval failure and idiosyncratic phonology generation or through lexical retrieval errors which are subsequently phonologically distorted (compound errors). However, further analysis of the phonological content of nonword errors argues against these interpretations. The phonological overlap between nonword errors and targets was compared to that expected by chance. Above chance level phonological accuracy (e.g. village, /lividz/) is unlikely without adequate access to the lexical representation of a word, whereas phonological accuracy at the chance level would occur following lexical error or lexical retrieval failure (Godbold et al., 2013; Olson et al., 2007; Robson et al., 2003). This is particularly the case in naming where only a lexical processing route is available. Although this analysis confirmed severe levels of impairment on average nonwords contained less than half of the targets phonemes (see Figure 2) - the 
phonological accuracy of nonword errors was above chance in all participants in almost all

408

409

410

411

412

413

414 tasks, supporting the hypothesis that accurate lexical information is available. This was further supported by analysis of the distribution of the POI of nonword errors. It has been proposed that a single phonological nonword error source will produce a normal distribution of phonological accuracy in nonwords whereas a dual lexical-phonological source will produce a bimodal distribution with a large proportion of errors with very limited target overlap (Olson et al., 2007; 2015; Schwartz et al., 2004). The majority of POI distributions in the current study adhered to a normal distribution or were negatively skewed, a trend also noted in existing Jargon case studies (Olson et al., 2007; 2015), suggesting that lexically mediated nonword errors were scarcely produced. In addition to these analyses, qualitative interpretation of participant data demonstrated little to no evidence of compound errors, i.e. moderate phonological disruption of semantic errors, hypothesised as reflecting a lack of lexical influence (Olson et al., 2015). Together these results do not indicate a significant lexical contribution to nonword errors in Jargon aphasia. Instead it is interpreted that greater phonological accuracy in reading and repetition than in naming indicates some ability to use input phonological information to support phonological encoding. This pattern is compatible with the earlier interpretation that tasks of repetition and reading can be accomplished either by lexico-phonological processing when word recognition has triggered at least partially correct phonological information, or nonlexical processing which maps input - output phonology, again, with some degree of success.

\subsection{Exception cases}

Observation of the case series highlighted a number of notable exceptions. Participant 4's nonword phonological accuracy in naming was not significantly different from chance, and the corresponding POI distribution was non-normally distributed. POI distribution normality 
432 violations also occurred for two other participants $-\mathrm{p} 9$ in naming, and p10 in naming and 433 repetition. It is possible that these individuals have more significant lexical processing impairment than the other participants and that this impairment contributed to nonword production. The existence of lexically mediated errors, possessing very limited accurate phonology, is expected to co-occur alongside a group of errors containing more moderate degrees of target phonology, together eliciting a bimodal accuracy distribution (Olson et al., 2007; 2015; Schwartz et al., 2004). Bimodal distributions were not observed in these participants. Instead, positively skewed histograms (see Figure 4) were observed, indicating that, for these particular individuals, nonword accuracy was heavily weighted towards lower accuracy production. This trend indicates very severe phonological encoding impairments, particularly in naming where no sub-lexical support was available. Participant 10 displayed a POI normality violation in repetition, alongside a low POI average score for this task $(0.27$, see Figure 3a). Individuals with Wernicke's aphasia have well documented auditory and input phonological processing impairments which are associated with their language comprehension impairment (Robson, et al., 2012; Robson, Pilkington, Evans, DeLuca, \& Keidel, 2017). Participant 10 displayed the most severe language comprehension impairment (Table 1), indicating considerable auditory processing difficulties and a reduced ability to use phonological input information to boost production in repetition via lexical or nonlexical processing.

\subsection{Jargon phonological inventories}

453 Although these three cases presented with the greatest degree of nonword production impairment, the majority of participants in the current study presented with severe Jargon aphasia. It has been proposed that such individuals may suffer from a distorted phonological system due to long standing nonword production warping phonological representations and 

explored by analysing the occurrence of phoneme segments within nonwords to determine whether nonword phoneme frequency distributions pertain to the typical phoneme distributions observed in English, thus indicating whether the phonological system in Jargon aphasia operates in line with its typical numerical distributional properties. All but one participant (p2) in the current study produced phonological segments in line with that expected in English, suggesting that, for the most part, the phonological system maintains its typical organisation and structure. This is contrary to results reported in previous studies, where evidence of idiosyncratic or default phonological useage is documented (Eaton et al., 2010; Moses et al., 2004). However, the current data were sampled at a single time point within what is typically a prolonged recovery trajectory, when the majority of the group were not classified as chronic. Therefore current results cannot exclude that long-standing nonword production in Jargon aphasia may self-reinforce deviant phonological useage and alter the rates at which specific phonological segments reside. For example, participants p5 and p8 are statistically borderline in how their phonological distribution adhered to the normal observed phoneme useage, and p4 demonstrates over representation of a phonological segment (see Figure 5), suggesting that their phonological selection may be in the early stages of distortion and may evolve into an idiosyncratic system. Therefore, longitudinal analyses may be more suited to investigating this hypothesis.

\section{Conclusion}

This study investigated the degree to which lexical impairment contributed to the production of nonword errors in Jargon aphasia by analysing the number and content of nonword errors produced during repetition, reading and naming in a case series of 10 individuals with neologistic production. Overall, the phonological inventories of the group adhered to English 
norms indicating that Jargon nonword production arises through a phonological system that maintains the typical phonological organisation and suggests that production is constrained by lexico-phonological processing. The phonological content of nonwords indicated that some accurate lexical information is available for the majority of individuals with Jargon aphasia during word production. However, impairments in lexical recognition and processing

487 lead to reliance on phonological information to support production, thereby increasing the 488 number of nonwords. Picture naming, which does not involve the presentation of 489 phonological material, maximises lexical processing which reduces the likelihood of 490 observing a nonword. These results demonstrate that tasks which maximise phonological 491 processing demands increase the amount of Jargon and indicate that Jargon nonword error 492 production is phonologically mediated. 
A2kerman, T., \& Ellis, A. W. (2007). Case study: Where do aphasic perseverations come from? 495 Aphasiology, 21(10-11), 1018-1038. doi: 10.1080/02687030701198361

Aq\$am, A. L., Patterson, K., Bozeat, S., \& Hodges, J. R. (2010). The Cambridge Semantic Memory 497 Test Battery: detection of semantic deficits in semantic dementia and Alzheimer's disease. 498 Neurocase, 16(3), 193-207. doi: 10.1080/13554790903405693

Bgars, B. J., Motley, M. T., \& MacKay, D. G. (1975). Output editing for lexical status in artificially 500 elicited slips of the tongue. Journal of Verbal Learning and Verbal Behavior, 14(4), 382-391. 501 doi: https://doi.org/10.1016/S0022-5371(75)80017-X

Boze, A. (2013). Phonological therapy in jargon aphasia: Effects on naming and neologisms. 503 International Journal of Language \& Communication Disorders, 48(5), 582-595. doi: 504 10.1111/1460-6984.12038

B0rekingham, H. W. (1990). Abstruse neologisms, retrieval deficits and the random generator. $J$ 506 Neurolinguistics, 5(2-3), 215-235. doi: http://dx.doi.org/10.1016/0911-6044(90)90012-N

Bozkingham, H. W. (1977). The Conduction Theory and Neologistic Jargon. Language and Speech, 508 Vol 20, Issue 2, pp. 174 - 184. https://doi.org/10.1177/002383097702000209

Borkingham, H. W. (1981). Chapter 3 - Where Do Neologisms Come From? A2 - BROWN, JASON 510 W Jargonaphasia (pp. 39-62), New York: Academic Press.

Bııkingham, H. W., \& Kertesz, A. (1976). Neologistic jargon aphasia. In R. Hoops \& Y. 512 Lebrun (Eds.), Neurolingsuistics. Amsterdam: Swets \& Zeitlinger.

B113terworth, B. (1979). Hesitation and the production of verbal paraphasias and neologisms in jargon 514 aphasia. Brain and Language, 8(2), 133-161. doi: http://dx.doi.org/10.1016/0093515 934X(79)90046-4

B116terworth, B. (1992). Disorders of phonological encoding. Cognition, 42(1), 261-286. doi: 517 http://dx.doi.org/10.1016/0010-0277(92)90045-J

B118terworth, B., Swallow, J., \& Grimston, M. (1981). Chapter 5 - Gestures and Lexical Processes in 519 Jargonaphasia A2 - BROWN, JASON W Jargonaphasia (pp. 113-124). New York:

520 Academic Press.

5Drbett, F., Jefferies, E., \& Lambon Ralph, M. A. (2008). The use of cueing to alleviate recurrent 522 verbal perseverations: Evidence from transcortical sensory aphasia. Aphasiology, 22(4), 363523 382. doi: 10.1080/02687030701415245 
584theart, M., Curtis, B., Atkinsm P., \& Haller, M. (1993). Models of reading aloud: Dual-route and 525 parallel-distributed-processing approaches. Psychol. Rev. 100, 589-608. doi: 10.1037/0033$526295 X .100 .4 .589$

Baxis, C. J. (2005). N-Watch: A program for deriving neighborhood size and other psycholinguistic 528 statistics. Behavior Research Methods, 37(1), 65-70. doi: 10.3758/BF03206399

5291, G. S., Schwartz, M. F., Martin, N., Saffran, E. M., \& Gagnon, D. A. (1997). Lexical access in 530 aphasic and nonaphasic speakers. Psychol Rev, 104(4), 801-838.

Bones, P. B. (1963). On the Statistics of Spoken English. The Journal of the Acoustical Society of 532 America, 35(6), 892-904. doi: doi:http://dx.doi.org/10.1121/1.1918622

EaBon, E., Marshall, J., \& Pring, T. (2010). Like deja vu all over again: Patterns of perseveration in 534 two people with jargon aphasia. Aphasiology, 24(9), 1017-1031. doi: Pii 918865637 $53510.1080 / 02687030903249343$

5676old, C., Robson, H., \& Bose, A. (2013). Patterns of Phonological Overlap between Non-word 537 Error and Target in Jargon Aphasia. Procedia - Social and Behavioral Sciences, 94, 28-29. 538 doi: http://dx.doi.org/10.1016/j.sbspro.2013.09.011

5b9drick, M., \& Blumstein, S. E. (2006). Cascading activation from phonological planning to 540 articulatory processes: Evidence from tongue twisters. Lang Cogn Process, 21(6), 649-683. 541 doi: 10.1080/01690960500181332

G420dglass, H., Kaplan, E., \& Barresi, B. (2001). Boston diagnostic aphasia examination (3rd ed.). 543 Philadelphia: Lippincott Williams \& Wilkins.

Bamley, J. R., Dell, G. S., Kay, J., \& Baron, R. (2004). Evidence for the involvement of a 545 nonlexical route in repetition of familiar words: a comparison of single and dual route 546 models of auditory word repetition. Cognitive neuropschology, 21(2/3/4) 147-158.

Kuttesz, A., \& Benson, D. F. (1970). Neologistic Jargon: A Clinicopathological Study. Cortex, 6(4), 548 362-386. doi: http://dx.doi.org/10.1016/S0010-9452(70)80002-8

K49hn, S. E., Smith, K. L., \& Alexander, M. P. (1996). Differential recovery from impairment to the 550 phonological lexicon. Brain and Language, 52(1), 129-149. doi: 10.1006/brln.1996.0007

\$barshall, J. (2006). Jargon aphasia: What have we learned? Aphasiology, 20(5), 387-410. doi: $552 \quad 10.1080 / 02687030500489946$

\$58ses, M. S., Nickels, L. A., \& Sheard, C. (2004). Disentangling the web: Neologistic perseverative 554 errors in jargon aphasia. Neurocase, 10(6), 452-461. doi: 10.1080/13554790490894057

\$boses, M. S., Nickels, L. A., \& Sheard, C. (2007). Chips, cheeks and carols: A review of recurrent 556 perseveration in speech production. Aphasiology, 21(10-11), 960-974. doi:

557 10.1080/02687030701198254 
Lerari, N., and Dell, G. (2013). How damaged brains repeat words: A computational approach. 559 Brain and Language. 126(3), 327-337. https://doi.org/10.1016/j.bandl.2013.07.005

Storari, N., Kitteridge, A. K., Dell, G. S., \& Schwartz, M. F., (2010). Naming and repeition in 561 aphasia: Steps, routes and frequency effects. Journal of Memory and Language, 1;63(4):541562 559. doi: 10.1016/j.jml.2010.08.001

563on, A., Halloran, E., \& Romani, C. (2015). Target/error overlap in jargonaphasia: The case for a 564 one-source model, lexical and non-lexical summation, and the special status of correct 565 responses. Cortex, 73, 158-179. doi: http://dx.doi.org/10.1016/j.cortex.2015.06.028

G6son, A. C., Romani, C., \& Halloran, L. (2007). Localizing the deficit in a case of jargonaphasia. 567 Cognitive Neuropsychology, 24(2), 211-238. doi: 10.1080/02643290601137017

Bä\&ington, E., Keidel, J., Kendrick, L. T., Saddy, J. D., Sage, K., \& Robson, H. (2017). Sources of 569 Phoneme Errors in Repetition: Perseverative, Neologistic, and Lesion Patterns in Jargon 570 Aphasia. Frontiers in Human Neuroscience, 11(225). doi: 10.3389/fnhum.2017.00225

Bøbson, H., Pilkington, E., Evans, L., DeLuca, V. and Keidel, J. (2017) Phonological and semantic 572 processing during comprehension in Wernicke's aphasia: a N400 and Phonological Mapping 573 Negativity study. Neuropsychologia, 100. pp. 144-154. ISSN 0028-3932 doi:

574 https://doi.org/10.1016/j.neuropsychologia.2017.04.012

Bæ\$\$son, H., Sage, K. and Lambon Ralph, M. A. (2012) Revealing and quantifying the impaired 576 phonological analysis underpinning impaired comprehension in Wernicke's aphasia.

577 Neuropsychologia, 50 (2). pp. 276-288. ISSN 0028-3932 doi:

578 https://doi.org/10.1016/j.neuropsychologia.2011.11.022

Bðゆson, J., Pring, T., Marshall, J., \& Chiat, S. (2003). Phoneme frequency effects in jargon aphasia: 580 a phonological investigation of nonword errors. Brain and Language, 85(1), 109-124.

R8zlofs, A. (2004). Seriality of phonological encoding in naming objects and reading their names. 582 Mem. Cognit. 32, 212-222. doi: 10.3758/BF03196853

S8liwartz, M. F., Wilshire, C. E., Gagnon, D. A., \& Polansky, M. (2004). Origins of nonword 584 phonological errors in aphasic picture naming. Cognitive Neuropsychology, 21(2-4), 159-186. 585 doi: 10.1080/02643290342000519

$\$ \&$ 6evitch, M. S. (2002). The Influence of Phonological Similarity Neighborhoods on Speech 587 Production. Journal of Experimental Psychology. Learning, Memory, and Cognition, 28(4), 588 735-747. doi: 10.1037//0278-7393.28.4.735 


\section{$590 \quad$ List of table titles}

591 Table 1: Demographic and Boston Diagnostic Aphasia Examination (BDAE) short form 592 percentile results.

593 Table 2: The number of correct responses, nonwords and other errors produced by each 594 participant across Repetition, Reading and Naming.

595 Table 3: $p$ statistic from Shapiro Wilk normality test of POI distribution.

596 Table 4: $Z$ statistic and $p$ value from Kolmogorov Smirnov two (KS2) test comparing 597 normative and individual nonword phoneme frequency distributions. 


\section{List of figure titles and legends}

600 Figure 1 Title: Nonword Production in Repetition, Reading and Naming.

601 Figure 1 Legend: Bar chart displays the mean number of nonword responses in each task.

602 Individual markers indicate participant nonword numbers.

603 Figure 2 Title: Phonological Overlap Index in Repetition, Reading and Naming

604 Figure 2 Legend: Bar chart displays mean Phonological Overlap Index (POI) of nonword 605 errors in each production task. Individual markers represent participant POI means.

606 Figure 3: Participant Phonological Overlap Index vs. Chance Phonological Overlap Index 607 nonwords produced in Repetition (A), Reading (B) and Picture Naming (C). Error bars 608 indicate $95 \%$ confidence intervals.

609 Figure 4: Phonological Overlap Index distributions when normality violated. (A) p10

610 Naming, (B) p10 Repetition, (C) p9 Naming, (D) p4 Naming.

611 Figure 5: Phoneme frequency distributions for English norms and participants. 
Disclosure statement: The authors declare that the research was conducted in the absence of any commercial or financial relationships that could be construed as a potential conflict of interest.

Acknowledgments: We would like to thank the ten participants and their families for all their hard work and dedication to this research.

Funding statement: This research was funded by a Stroke Association Postgraduate Fellowship awarded to Emma Pilkington (TSA PGF 2015-02) and a Stroke Association Senior Research Training Fellowship awarded to Holly Robson (TSA SRTF 2012/02). 